\title{
Light emitting diodes (LEDs) applied to microalgal production
}

\author{
Peter S.C. Schulze ${ }^{1,2}$, Luísa A. Barreira ${ }^{1}$, Hugo G.C. Pereira ${ }^{1}$, \\ José A. Perales ${ }^{2}$, and João C.S. Varela ${ }^{1}$ \\ ${ }^{1}$ Centre of Marine Sciences, University of Algarve, Campus de Gambelas, 8005-139 Faro, Portugal \\ ${ }^{2}$ Centro Andaluz de Ciencia y Tecnología Marinas, University of Cadiz, Campus Universitario de Puerto Real, Cádiz, Spain
}

\begin{abstract}
Light-emitting diodes (LEDs) will become one of the world's most important light sources and their integration in microalgal production systems (photobioreactors) needs to be considered. LEDs can improve the quality and quantity of microalgal biomass when applied during specific growth phases. However, microalgae need a balanced mix of wavelengths for normal growth, and respond to light differently according to the pigments acquired or lost during their evolutionary history. This review highlights recently published results on the effect of LEDs on microalgal physiology and biochemistry and how this knowledge can be applied in selecting different LEDs with specific technical properties for regulating biomass production by microalgae belonging to diverse taxonomic groups.
\end{abstract}

\section{Light in microalgal production}

Microalgal biomass is used as feed in aquaculture, bulk food, and as feedstock for food or feed supplements, nutraceuticals, and cosmetics, and has been considered as a promising feedstock for biofuel production [1,2]. Photoautotrophic growth of microalgae requires $\mathrm{CO}_{2}$, a growth medium containing nutrients, such as nitrogen and phosphorus, and a light source. Under heterotrophic and mixotrophic conditions, many microalgal species are able to use organic matter as a source of carbon and other nutrients. Photosynthesis in photoautotrophic and mixotrophic microalgae can be driven by sunlight or artificial light. Although sunlight is the most cost-effective energy source for microalgal production, artificial light is still economically feasible when biomass is used as a feedstock for highvalue products, such as food or feed supplements (e.g., carotenoids and n-3 polyunsaturated fatty acids) or nutraceuticals [1]. Artificial light also provides better regulation of the photosynthetic photon flux density (PPFD; see Glossary), photoperiod, and light spectra in microalgal production, which can result in gains in biomass productivity and quality, two key factors for the success of any agricultural or industrial product [2]. However, the use of artificial light sources comes at a cost, so their

Corresponding author: Varela, J.C.S. (jvarela@ualg.pt).

Keywords: microalgae; light-emitting diodes (LEDs); light requirements; energy efficiency; microalgal biomass; microalgal biochemicals.

0167-7799/

(C) 2014 Elsevier Ltd. All rights reserved. http://dx.doi.org/10.1016/j.tibtech.2014.06.001 improvement in terms of photosynthetic and electronic efficiency can provide a wider and cheaper array of products obtained from microalgal biomass [2]. This strategy has already been recognized as useful for horticulture [3] .

Artificial lighting in microalgal research and production usually involves fluorescence lamps (FLs), which have wide emission spectra, including wavelengths with low photosynthetic activity for certain microalgae [2]. Alternatively, light-emitting diodes (LEDs) can be used [1,3,4]. LEDs are long-lasting ( $\sim 50000 \mathrm{~h})$, mercury-free, and fastresponding (nanosecond scale) artificial light sources emitting nearly monochromatic light at various wavelengths by virtue of solid-state electronics [3,4]. Hence, LEDs can also be applied to adjust the biochemical composition of the biomass produced by microalgae via single wavelengths at

\section{Glossary}

Color rendering index (CRI): indicates how true the color of an irradiated object is revealed by a particular light source to human eyes.

Color temperature (K): denotes the trend of a light spectrum towards bluish or reddish wavelengths and is related to the irradiation spectrum of a heated Planck's black body at a given temperature (K). The human eye perceives a black body heated to $2000 \mathrm{~K}$ as having a reddish tint, whereas at $10000 \mathrm{~K}$ a black body has a more bluish appearance.

Luminous efficacy $(\mathrm{Im} / \mathrm{W})$ : efficiency of electrical power transformation to optical energy as perceived by the human eye. This measure is commonly used to compare light sources. The absorption spectrum of light-harvesting complexes of photosynthetic organisms [11] may differ from that of the human eye [4], and thus luminous efficacy is not a suitable indicator of the quality of a light source for photoautotrophic cultivation. Light sources with the same wattage and the same PCE can have very different luminous efficacy, depending on their spectrum. For conversion of luminous efficacy to $\mu \mathrm{mol}$ photons $\mathrm{s}^{-1} \mathrm{~W}^{-1}$, see [1].

Photosynthetically active photon flux density (PPFD): amount of photosynthetic active photons in $\mu \mathrm{mol}$ striking a surface of $1 \mathrm{~m}^{2}$ in $1 \mathrm{~s}$. The photosynthetically active wavelength range is $400-700 \mathrm{~nm}$. However, algae and plants can be photosynthetically active between 380 and $750 \mathrm{~nm}$. Measurement of the PPFD of light sources with narrow spectra using quantum sensors often causes significant over- or underestimation at certain wavelengths, and correction factors are essential $[70,71]$.

Power conversion efficiency (PCE): ratio between the electrical optical output and input energy ( $W_{\text {output(optical) }} / W_{\text {input }}$ ) of a illuminant as a percentage. When the absolute spectral irradiance $\left(\mathrm{W} \mathrm{m} \mathrm{m}^{-2} \mathrm{~nm}^{-1}\right.$; obtained by radiometric measurements, for example) of an illuminant is known, PCE can be used to estimate the photon flux and photosynthetic efficiency.

White light: light composed of photons of different wavelengths among a broadband spectrum. It is usually evaluated in the human vision context via parameters such as the color temperature, color rendering index, or luminous efficacy.

$\boldsymbol{\lambda}_{\mathrm{a}}$ and $\boldsymbol{\lambda}_{\boldsymbol{e}}$ : maximum absorption and emission wavelengths, respectively. $\lambda_{a_{-} \text {red }}$ and $\lambda_{a_{-} \text {blue }}$ are absorption peaks within the red and blue light ranges, respectively. $\lambda_{\max }$ denotes the preferred wavelength for optimal growth (see Table S1 in the supplementary material online) or preferred wavelength for a metabolic effect (see Table 1 in the main text), whereas $\lambda_{\min }$ denotes an alternative preferred wavelength that may be suboptimal. 


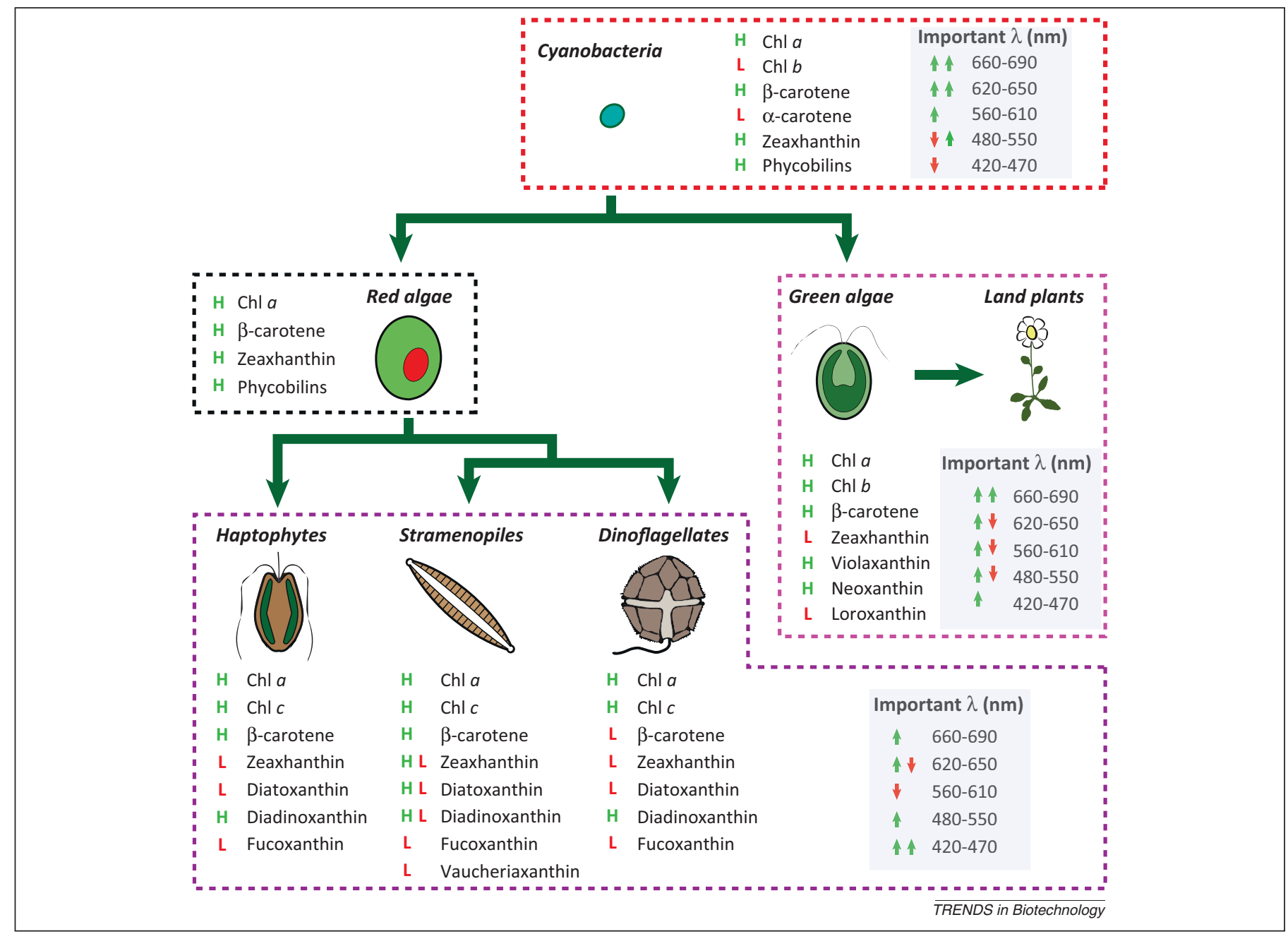

Figure 1. Approximate light requirements of microalgae according to results in Table $\mathrm{S} 1$ in the supplementary material, main pigments, and the evolutionary relationships among major microalgal megagroups [12-14]. Abbreviations: Chl, chlorophyll; $\mathrm{H}$, high pigment content; L, low pigment content; $\mathrm{H}$ L, variation between high and low pigment contents among species.

different light intensities or pulse light frequencies [5-9]. Exposure of microalgae growing in photobioreactors to different light sources and the economic feasibility of this approach have been discussed previously $[1,2,10,11]$. However, the use of LEDs to supply specific light ranges for microalgal growth and to obtain high-value biochemical traits has not been reviewed extensively. LEDs will certainly become one of the world's most important light sources [4], so this review provides an overview of the application of various LED types to microalgal production.

\section{Effects of light quality on algal growth}

The number of photons at blue or red wavelengths that can be captured by a molecule of chlorophyll in an alga depends on the cellular architecture, pigment composition, and chloroplast arrangement. Interestingly, the evolutionary history of microalgae as purported by Keeling [12] seems to account for the preference of microalgae to grow under either blue $(\lambda \approx 420-470 \mathrm{~nm})$ or red $(\lambda \approx 660 \mathrm{~nm})$ light (Table S1 in the supplementary material online). This preference correlates well with the evolutionary megagroup of each microalga, which in turn seems to reflect the pigment composition of the light-harvesting complexes in their chloroplasts (Table S1 in the supplementary material online) [13]. As discussed by Keeling [12], a primary endosymbiotic event between a eukaryote and a chlorophyll-b-containing ancestor of cyanobacteria gave rise directly or indirectly to most photoautotrophic eukaryotes (Figure 1). Cyanobacteria, especially those lacking chlorophyll $b$, use chlorophyll $a\left(\lambda_{\mathrm{a}} \approx 430\right.$ and $\left.680 \mathrm{~nm}\right)$ and accessory phycobiliproteins such as phycoerythrin $\left(\lambda_{\mathrm{a}} \approx\right.$ $550 \mathrm{~nm})$ and phycocyanin $\left(\lambda_{\mathrm{a}} \approx 620 \mathrm{~nm}\right)$, in light-harvesting protein-pigment (LHP) complexes [14]. As a result, cyanobacteria are able to utilize mostly red, yellow, and green light and, to a significantly lesser extent, blue light $[12,15,16]$.

The first endosymbiotic event with the ancestor of cyanobacteria as the endosymbiont led to the appearance of chlorophytes (green algae) and rhodophytes (red algae) [17]. Red light is crucial for the growth of chlorophytes (and land plants) [13]. However, these organisms are able to utilize blue light more efficiently than cyanobacteria are, probably because of the loss of chlorophyll $b$ by many cyanobacterial species [14] and a higher diversity of carotenoids in photosynthetic eukaryotes (Figure 1). As a trade-off, chlorophytes lack the ability to utilize yellow and green light extensively because they lost phycobilins during evolution [14]. Therefore, for chlorophyll-acontaining microalgae, major wavelengths are within the range $420-470 \mathrm{~nm}$ and/or $660-680 \mathrm{~nm}$, and accessory 
wavelengths are located below, between, or above these ranges. Exposure of microalgae to accessory wavelengths $\left(\lambda_{\mathrm{e}} \approx 500-630 \mathrm{~nm}\right)$ alone consistently leads to lower biomass production compared to growth under either blue $\left(\lambda_{\mathrm{e}} \approx 430-470 \mathrm{~nm}\right)$ or $\operatorname{red}\left(\lambda_{\mathrm{e}} \approx 660 \mathrm{~nm}\right)$ LEDs $[6,12,15,18-24]$. Green $\left(\lambda_{\mathrm{e}} \approx 525-550 \mathrm{~nm}\right)$ LEDs were often found to be highly unsuitable for microalgae if used without additional light sources (Table $\mathrm{S} 1$ in the supplementary material online) $[1,22,23,25,26]$.

Secondary endosymbiotic events involving heterotrophic eukaryotes and green algae gave rise to mixotrophic euglenids and chlorarachniophytes with a pigment composition and most probably light requirements similar to those of the ancestors of their plastids. Conversely, microalgae such as rhodophytes and glaucophytes are probably better adapted to shorter wavelengths (blue, green, yellow) than chlorophytes are because they retained phycobilins during evolution [12].

Cryptomonads and haptophytes (e.g., Isochrysis), belonging to the Hacrobia megagroup, and heterokontophytes (e.g., diatoms) and dinoflagellates, belonging to the Stramenopiles-Alveolata-Rhizaria (SAR) megagroup, are assumed to have evolved from secondary endosymbiosis of a heterotrophic eukaryote engulfing a rhodophyte, whereupon most species lost phycobilins. Instead, they developed a higher diversity of carotenoids and acquired chlorophyll $c$, a type of chlorophyll that absorbs strongly in the blue light range [27]. As a result, Hacrobia and SAR algae are usually better equipped for using bluish light compared to cyanobacteria and chlorophytes (Table S1 in the supplementary material online). Serial and tertiary endosymbiosis resulted in other eukaryotic photoheterotrophs (mostly dinoflagellates) with preferred wavelengths probably similar to those of the ancestors of their endosymbionts, although no studies on the light requirements of these microalgae have been found. Therefore, a shared evolutionary history of microalgae as suggested by Keeling's tree of endosymbiotic events [12] may provide information about the light requirements of algae within groups (Figure 1). For further information about pigments and their role in light harvesting for photosynthesis, see work by Cheng and Fleming [28] and Scholes et al. [29].

\section{Effects of light quality on microalgal traits}

In general, photons with a wavelength of $660-680 \mathrm{~nm}$ yield the highest quantum efficiencies in most plants and algae containing chlorophyll $a\left(\lambda_{\mathrm{a} \text { red }}=680 \mathrm{~nm}\right)$ and/or $b\left(\lambda_{\mathrm{a} \_\mathrm{r}-}\right.$ ed $=660 \mathrm{~nm})[1,11]$. The efficiency sharply decreases at longer wavelengths [30,31]. Interestingly, red to far-red light $(\lambda \approx 630-750 \mathrm{~nm})$ induces high growth rates and smaller cells by accelerating the cell cycle in many microalgae of diverse evolutionary lines $[1,16,19,32-35]$. However, far-red light can suppress volumetric biomass production when supplementing a broadband light source [34] because it regulates light-harvesting mechanisms in microalgae [35]. In terrestrial plants, far-red light promotes flowering, fruit development, and biomass production, but it can also cause growth arrest due to a complete breakdown of photosynthesis if not applied properly [36].

Red to far-red light can be detected by photoreceptors such as phytochromes in land plants and charophytes, as well as multiple light-sensing photoreceptors in cyanobacteria $[37,38]$. In free-swimming chlorophytes, no obvious genes encoding phytochromes have been found. Instead, an "animal-like" cryptochrome seems to be the long-soughtafter red receptor in chlorophytes, although it is unable to detect far-red light [39]. This multitude of red/far-red photoreceptors in different evolutionary lines strongly indicates that caution must be used when extrapolating how the red or far-red light range is detected and how it regulates growth. Clearly, further research is needed to understand how different photosynthetic organisms detect and respond to this range of the electromagnetic spectrum.

For shorter wavelengths, blue light influences gene expression and several metabolic pathways in algae and plants via photoreceptors such as cryptochromes, phototropins, aureochromes, and neochromes [38-42]. Blue light is, for example, responsible for endogenous breakdown of carbohydrate reserves [43], which may explain why the haptophyte Isochrysis sp. T-ISO exposed to blue light and grown in a chemostat displayed lower carbohydrate content than when grown under other wavelengths [44]. However, the opposite or no significant effect has been obtained in green algae grown in batch culture [23,24]. Kim et al. found that changing the ratio between red and blue light affected nutrient utilization more than biomass production [22] because of increased enzyme activity such as carbonic anhydrase activation for hydrogen carbonate $\left(\mathrm{HCO}_{3}{ }^{-}\right)$uptake [45] or nutrient uptake due to activation of reductases for nitrite, nitrate, and phosphorus utilization $[21,43,45,46]$. Blue light, most probably via photoreceptors such as phototropins, seems to induce pigment accumulation in several species (Table 1) $[7,26,39,46]$. Moreover, because the energy of blue photons is higher than that required for photosynthesis [10], blue light might result in nonphotosynthetic quenching (NPQ), generating reactive oxygen species (ROS) [7]. Therefore, to protect the photosynthetic apparatus against ROS, algae and plants accumulate photoprotective pigments (e.g., xanthophylls) $[3,7,11,47,48]$.

Lastly, increased green light content within a broadband light source can increase leaf photosynthesis and biomass production in land plants $[3,49,50]$ because these photons can penetrate through the canopy and promote photosynthesis in leaves located below [12,16,51]. Mohsenpour and Willoughby demonstrated that green light could induce pigmentation in algae [51]. However, further research is needed before drawing a definitive conclusion about the effect of green light on algae.

\section{LEDs for microalgal production}

Light quality influences the growth and biochemistry of microalgae, so artificial lighting can be used to manipulate the final biomass for specific uses, particularly for high-end markets. To design an artificial lighting system for microalgal growth, the electronic and photosynthetic efficiency of FLs and LEDs must be considered. On conversion of an electric current to light in LEDs (Box 1) and FLs (Box 2), energy losses occur because of thermal dissipation and inward light reflection and reabsorption, among other factors. Reduction of such inefficiency has resulted in higher power conversion efficiency (PCE) for LEDs 
Table 1. Light quality effects on microalgal biochemical composition at specific wavelengths

\begin{tabular}{|c|c|c|c|}
\hline Light & $\lambda_{\max }(\mathrm{nm})$ & Alga & Effects \\
\hline \multirow[t]{13}{*}{ Blue } & $460-475$ & $\begin{array}{l}\text { Arthrospira platensis } \\
\text { (syn. Spirulina platensis) }\end{array}$ & $\begin{array}{l}\text { Lowest chl and phycocyanin content in biomass compared to yellow, } \\
\text { green, red, and white LEDs }\end{array}$ \\
\hline & $440-470$ & Chlorella sp. & Higher lipid content in biomass compared to red $(650-680 \mathrm{~nm})$ LEDs \\
\hline & 500 & Chlorella sp. & Blue light induces slightly higher lipid production compared to red light \\
\hline & 470 & Dunaliella salina & $\begin{array}{l}\beta \text {-Carotene and lutein accumulation when blue light was supplemented } \\
\text { with red }(660 \mathrm{~nm})\end{array}$ \\
\hline & 470 & Haematococcus pluvialis & Accumulation of red pigments \\
\hline & $380-470$ & Haematococcus pluvialis & Astaxanthin accumulation \\
\hline & NA & Isochrysis T-ISO & $\begin{array}{l}\text { Higher protein content and lower carbohydrate and chl content per cell } \\
\text { compared to white FLs }\end{array}$ \\
\hline & NA & Isochrysis galbana & $\begin{array}{l}\text { Higher DHA and phospholipid content compared to red LEDs under } \\
\text { intermittent light }(f=10 \mathrm{kHz})\end{array}$ \\
\hline & 475 & Nannochloropsis oceanica CY2f & $\begin{array}{l}\text { Blue and red }\left(\lambda_{\mathrm{e}} \sim 630 \mathrm{~nm}\right) \text { LEDs showed highest EPA content in biomass } \\
\text { compared to FLs and white and yellow LEDs }\end{array}$ \\
\hline & 470 & Nannochloropsis sp. & $\begin{array}{l}\text { Highest palmitoleic acid and lowest EPA content compared to red, green, } \\
\text { and white LEDs under phototrophic conditions; highest total FAMEs per } \\
\text { dry weight under blue, green, and white light, lowest under red when } \\
\text { grown mixotrophically; similar FAME contents under phototrophic } \\
\text { conditions }\end{array}$ \\
\hline & 450 & Nitzschia sp. & Highest chl content compared to red $(650 \mathrm{~nm})$ and yellow ( $590 \mathrm{~nm}$ ) LEDs \\
\hline & NA & Phaeodactylum tricornutum & $\begin{array}{l}\text { Lager pool of xanthophyll cycle pigments and higher chl a content } \\
\text { compared to red and white LEDs (low light conditions) }\end{array}$ \\
\hline & 470 & Tetraselmis suecica F\&M-M33 & $\begin{array}{l}\text { Higher chl accumulation compared to cool white FLs and red, green, and } \\
\text { blue LEDs; higher carbohydrate content for cells grown under blue } \\
\text { compared to red LEDs }\end{array}$ \\
\hline \multirow[t]{2}{*}{ Green } & NA & Chlorella vulgaris & $\begin{array}{l}\text { Higher chl accumulation compared to blue, yellow, orange, and red } \\
\text { broadband light spectra }\end{array}$ \\
\hline & 550 & Nannochloropsis sp. & $\begin{array}{l}\text { Higher AA content under phototrophic conditions compared to FLs and } \\
\text { red and white LEDs }\end{array}$ \\
\hline \multirow[t]{5}{*}{ Red } & 660 & Botryococcus braunii Bot-144 & $\begin{array}{l}\text { Evidence of higher carotenoid/chlorophyll ratio compared to blue and } \\
\text { green LEDs }\end{array}$ \\
\hline & 660 & Chlorella sp. & Highest biogas production compared to yellow, blue, and white LEDs \\
\hline & 660 & $\begin{array}{l}\text { Mychonastes homosphaera } \\
\text { (syn. Chlorella minutissima) }\end{array}$ & $\begin{array}{l}\text { Increased C18:2 and decreased C18:3 content in FAME; no fatty acid } \\
\text { changes between FLs and white LEDs; total FAMEs unaffected among all } \\
\text { light sources }\end{array}$ \\
\hline & 680 & Nannochloropsis sp. & $\begin{array}{l}\text { Higher oleic acid content compared to blue, green, and white LEDs under } \\
\text { phototrophic conditions }\end{array}$ \\
\hline & NA & Tetraselmis suecica F\&M-M33 & $\begin{array}{l}\text { EPA content increased under red light compared to blue, green, and } \\
\text { white LEDs }\end{array}$ \\
\hline Far-red & NA & Dunaliella salina (syn. D. bardawil) & Higher carotenoid levels compared to cells grown under FLs \\
\hline
\end{tabular}

Abbreviations: AA, arachidonic acid; chl, chlorophyll; EPA, eicosapentaenoic acid; FAME, fatty acid methyl ester; FL, fluorescent lamp; NA, spectrum not available or broadband spectrum.

(up to 50\%) [4] compared to gas-discharge lighting technologies such as FLs ( $30 \%)$ (Table S2 in the supplementary material online) $[52,53]$.

At present, cool white phosphor-converted-LEDs (pc-LEDs) yield the highest PPFD per input wattage (PPFD/W) among white LEDs and can be highly photosynthetically active because of their blue emission peak $\left(\lambda_{\mathrm{e}} \approx\right.$ 440-460 nm) [54], which almost perfectly fits the blue absorption spectrum of LHP in many plants and algae $[11,55,56]$. Both FLs and pc-LEDs emit light within the range of photosynthetically important long wavelengths $\left(\lambda_{\mathrm{e}}>650 \mathrm{~nm}\right)$, whereas most color-mixed-LEDs (cm-LEDs) display a sharp decrease in light emission at shorter wavelengths [4]. Furthermore, white pc-LEDs and FLs emit a broad spectrum of light with high photon release in the blue and green spectral ranges that decreases at $\lambda_{\mathrm{e}}>650 \mathrm{~nm}$. pcLEDs and FLs have similar emission spectra, so pc-LEDs might be well suited for FL replacement within the same color temperature range without causing significant changes in algal growth rates and biochemical properties.
This replacement would lead to more competitive energy usage for biomass production (Table S2 in the supplementary material online) $[1,55,57]$. Nevertheless, unlike FLs or pc-LEDs, single-color LEDs usually have higher PCE because they can emit at specific wavelengths without the use of phosphor layers and thus avoid losses ( $>30 \%)$ for conversion of higher- to lower-energy photons [4]. However, singlecolor LEDs emitting within the green-amber light range often have very low PCE, a problem known as the green gap or green-yellow gap [58].

In addition, more photons are usually released by LEDs emitting at longer wavelengths (e.g., red), resulting in higher PPFD/W ratios than for LEDs emitting at shorter wavelengths (e.g., blue) [1], because blue photons are more energetic than red photons. Specifically, red $\left(\lambda_{\mathrm{e}}=660 \mathrm{~nm}\right)$ LEDs can emit double the number of photons that blue LEDs emit, whereas green LEDs emit approximately three times fewer photons than red LEDs do [5,18,58-60]. At face value, these results suggest that $660-\mathrm{nm}$ red LEDs are able to sustain biomass growth with the highest energy 


\section{Box 1. How LEDs work}

LEDs are semiconductor devices consisting of a positive ( $P$ doped) layer and a negative ( $\mathrm{N}$ doped) layer (Figure IA). The $\mathrm{P}$ layer has an excess of electron holes in the valence band because of the presence of acceptor dopant atoms; the $\mathrm{N}$ layer has excess electrons in the conduction band owing to the presence of donor dopant atoms. When $\mathrm{N}$ and $\mathrm{P}$ semiconductors are brought together, excess carriers diffuse to the opposite side, resulting in a depletion region without free carriers. Application of an opposite voltage allows electrons from the $\mathrm{N}$ side and holes from the $\mathrm{P}$ side to enter the depletion region and recombine. This recombination corresponds to deexcitation of an electron from the conduction band to the valence band, and a photon with the corresponding difference in energy (energy gap between the bands) can be released. A blue-green
(365-550 $\mathrm{nm})$ InGaN semiconductor has a wider energy gap than an orange-red (560-650 nm) emitting AIGalnP diode or a red-infrared $(630-940 \mathrm{~nm})$ GaAlAs chip (Figure IB). Thus, recombination of semiconductor materials controls the wavelengths (color and energy of emitted light). To increase the efficiency, most (high-power) LED chips are built up in heterostructures with a more complex internal structure comprising more than one semiconductor material, which can also include multiple quantum wells. Further information on the theoretical background and application of these techniques is available in the literature $[52,54,75,76]$. White LEDs can be obtained by combining different LED chips in color-mixed LEDs (cm-LEDs) or by coating single blue chips with a photon-converting layer in phosphor-converted LEDs (pc-LEDs) [4].
(A)

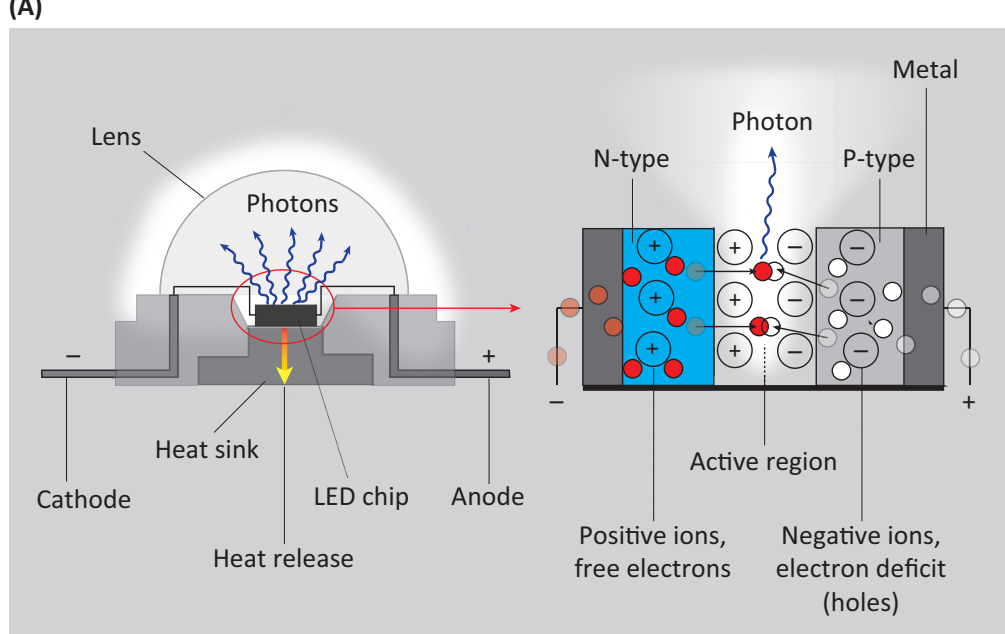

(B)

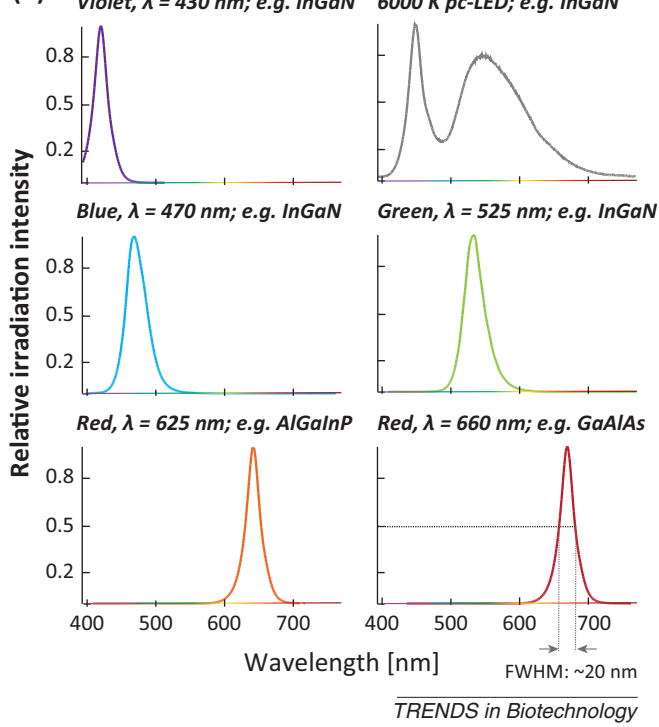

Figure I. (A) Simplified diagram showing how low-power (homostructured) LEDs work. (B) Emission spectra for different LEDs. The full width at half-maximum (FWHM) corresponds to the wavelength difference (usually $20 \mathrm{~nm}$ ) for which the LED attains $50 \%$ of its maximum intensity.

efficiency [1]. However, LEDs with $\lambda_{\mathrm{e}}>680 \mathrm{~nm}$ also release photons with photosynthetically inefficient wavelengths $\left(\lambda_{\mathrm{e}}>695 \mathrm{~nm}\right)$ [31,61], leading to lower photon utilization by the algae and thus less biomass production per input wattage. Nevertheless, caution is needed because PPFD/W ratios and PCE may vary with the LED manufacturer. Finally, the fast response time of LEDs compared to that of FLs can also be beneficial, because LEDs can be used to grow algae under customized flashing light via pulse width modulation (PWM; Box 3), which increases biomass production $[8,62,63]$ and might allow algae to exceed the proposed maximum photosynthetic efficiency of $17 \%$ [1].

\section{Tailored light sources}

\section{Spectral matching}

To maximize photosynthetic efficiency, all photons released from a light source should be captured by the photosynthetic apparatus of microalgae. A strategy to achieve a high level of light utilization is complete spectral matching of a light source to the photosynthetically active spectrum (PAS). However, this strategy can only yield an approximation because PAS measurement for microalgae is still difficult [30]. Determination of a general PAS for single taxonomic groups or megagroups, as done for green algae [64], may help to estimate the light quality required for a species belonging to a specific taxon. Absorption spectra of intact cells, however, are easier to obtain and may give a rough idea of the light quality needed for optimal growth. Comparison of the wavelengths of absorption peaks $[65,66]$ and the preferred wavelengths $\left(\lambda_{\max }\right.$ and $\lambda_{\text {min }}$ ) for growth (Table S1 in the supplementary material online) of algae within a given megagroup reveals a relatively good spectral match. However, the wavelength of the highest absorption peak seldom matches the preferred wavelength for optimal growth (either 420-470 or 660$670 \mathrm{~nm}$ ) of cyanobacteria and green algae. These species usually show better growth and biomass production under 660-nm LEDs (Table S1 in the supplementary material online), whereas maximum absorption often occurs in the blue range of the electromagnetic spectrum [65,66]. This is because the absorption spectra of cells include the contributions of all cellular components able to absorb or scatter light, which may not necessarily contribute to the lightharvesting processes needed for photosynthesis and thus may mask the true light requirements for growth of a specific microalga. 


\section{Box 2. How fluorescent lights work}

Electrodes located at each side of an FL are heated to induce emission of electrons into the space in front of the electrodes in a process called thermionic emission (Figure IA) [53]. An electric field is thereby built up and accelerates the electrons until their energy is high enough to excite gaseous mercury atoms, distributed in the space between the electrodes, from the ground stage to a higher energetic level. An excited mercury atom falls back to its ground stage and releases the energy difference by emitting a photon with energy of $\sim 5.5 \mathrm{eV}\left(8.83 \times 10^{-19} \mathrm{~J}\right)$. This high-energy UV photon can then be absorbed by the phosphor coating on the inside of the tube and is transformed into less energetic visible and photosynthetically active light $(380-750 \mathrm{~nm}$ or $1.7-3.2 \mathrm{eV}$ ).
The energy difference $(40-70 \%)$ is dissipated as heat, which drastically limits the FL efficiency [53]. Figure IB shows emission spectra for different FL types. The spectra of grow-light FLs have high levels of red light $(630-680 \mathrm{~nm})$ matching the chlorophyll $a$ and $b$ absorption peaks within the red spectral range. Therefore, these FLs can be used in growing photosynthetic organisms, although they are more expensive and less energetically efficient than FLs with major emission at shorter wavelengths (e.g., cool daylight FLs) [10]. The spectra of warm white FLs may not be suitable for growing algae in an energy-efficient manner because a significant proportion of their emission lies outside the major photosynthetic ranges (420-450 and 630-690 nm).
(A)

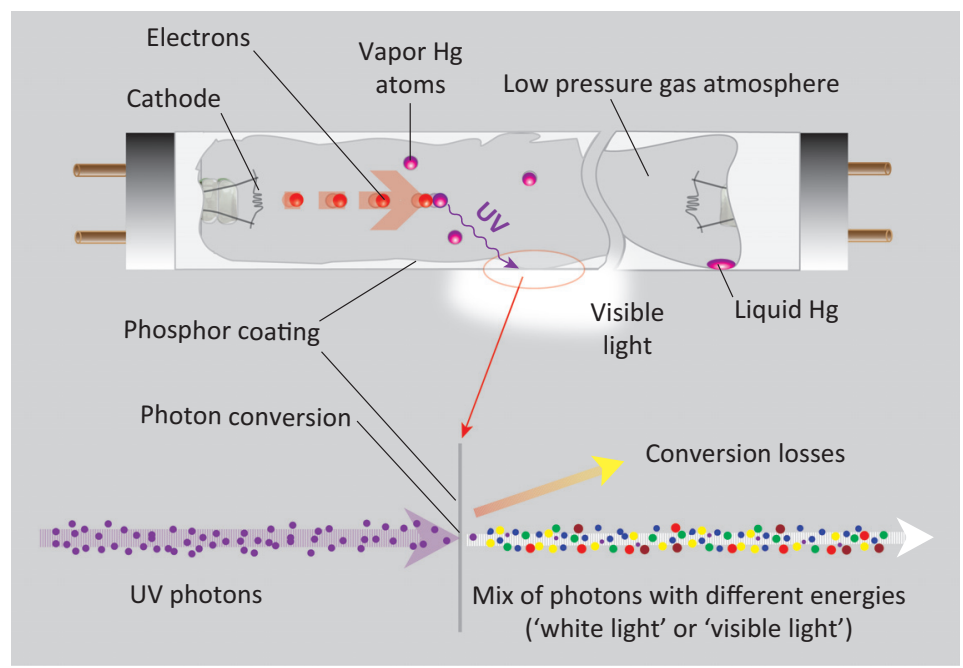

(B)

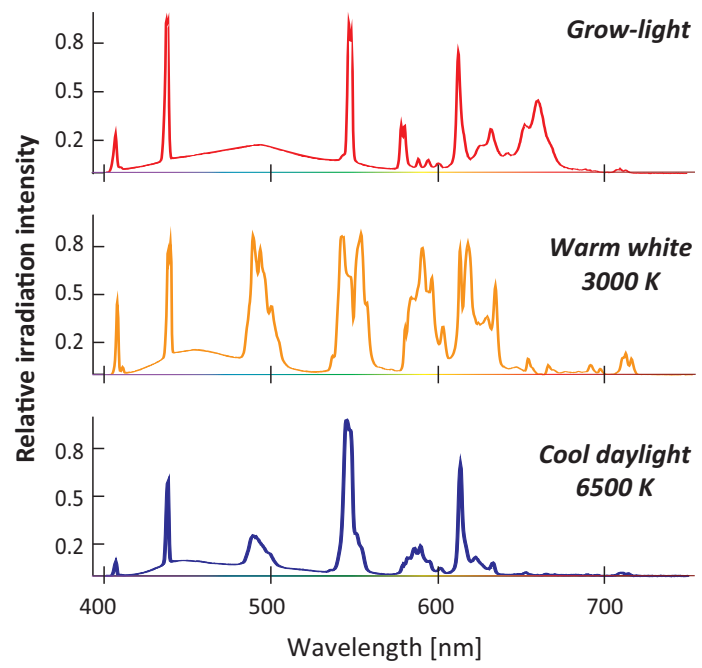

$\overline{\text { TRENDS in Biotechnology }}$

Figure I. (A) Simplified diagram showing how fluorescent lamps (FLs) work. (B) Emission spectra for different FL types, including grow-lights, warm white FL, and cold daylight FLs.

\section{Light quality and design of LED usage}

A tailored LED-based light source for high-volume production at the present state of the art might include pc-LEDs as a good starting point because their broadband phosphor emission at $\lambda_{\mathrm{e}} \approx 560 \mathrm{~nm}$ (Box 1) covers the green, yellow, and amber $(500-610 \mathrm{~nm})$ wavelength ranges. Violet-blue (preferably $420-450 \mathrm{~nm}$ ) and red $(660-670 \mathrm{~nm}$ ) wavelengths can then be further adjusted to the species selected for cultivation. However, white pc-LEDs, especially those with cooler color temperatures, also emit photons with $\lambda_{\mathrm{e} \text { blue }} \approx 440-460 \mathrm{~nm}$ (Box 1), so the levels of blue light available are higher. Moreover, as previously discussed, the taxonomy of the selected microalga may be used to predict the most important LEDs for growth (Figure 1 and Table S1 in the supplementary material online) or production of specific biochemicals (Table 1). Concerning red-toblue ratios, land plants showed optimal biomass production when red LED light was supplemented with $10-30 \%$ blue light [67]. This suggests that similar red and blue ratios may also be suitable for green algae, because their plastids are closely related to those of terrestrial plants in terms of structure, metabolism, and biochemical composition. This assumption seems to be correct, because mixing of red and blue photons in this proportion has often increased biomass production compared to red light alone $[7,21,60,68]$. Regarding other taxa such as cyanobacteria and SAR microalgae, higher blue light content may be needed. However, a relative dearth of studies on red-toblue ratios for these species means that no final conclusion is possible.

The success of increasing biomass productivity via tailored supplemental artificial light depends on environmental parameters for photosynthesis such as PPFD, light path length, $\mathrm{CO}_{2}$ concentration, $\mathrm{pH}$, macro- and micronutrient availability, and temperature, among other factors $[2,35]$. For example, nitrogen starvation can cause chromatic adaptation of cyanobacteria and red algae, resulting in degradation of phycobilisomes (phycobilin-containing light-harvesting complexes) and leading to diminished green light absorption [35]. Furthermore, the light path length of the bioreactor can influence the choice of wavelengths coming from a light source. A higher proportion of green-amber wavelengths might be beneficial for green and SAR algae grown in photobioreactors with a long light path or high-density cultures because photon absorption is lower, so they can travel deeper into the culture. In turn, the same light quality might be unsuitable for algae growing in flat panel photobioreactors with shorter light paths or low-density cultures. Lastly, Miao et al. concluded that the light saturation point for Skeletonema costatum is lower for a suitable than for an unsuitable light source [69]. If this result is confirmed in other species, growth of 


\section{Box 3. Dimming of LEDs}

The PPFD of LEDs can be controlled by PWM or AM, also referred to as continuous current reduction (CCR) [77,78]. PWM entails the use of alternating states in which the current is completely turned either on or off. At a fixed frequency, the relative duration of the on-period (duty cycle) can be regulated. A low duty cycle of $10 \%$ means that the LED is on for $10 \%$ of the on-and-off cycle. Alternatively, AM is achieved by reducing the current intensity, which in turn leads to decreased light output $[77,78]$. The luminous efficacy of the two dimming methods varies, though AM can reach $200 \%$ of the nominal efficacy of an LED provided by the supplier when dimmed to $10 \%$ of its nominal current. Conversely, at the same dimming level, PWM provides only $70 \%$ of the nominal efficacy [77]. AM dimming can be used to prevent photoinhibition during early growth stages because photons are emitted continuously over time at low PPFD. By contrast, PWMdimmed LEDs emit almost all photons within the LED capacity in a short duty cycle of flashing light, during which microalgae can be exposed to the fully available PPFD [8]. Because photosynthesis does not require continuous lighting $[8,62]$ and microalgae can display satisfactory photosynthetic rates even if the duration of the dark cycle is increased [62], PWM may be used as an effective tool for controlling microalgal growth. In addition, an increase in the instantaneous maximum PPFD within the duty cycle of flashing LEDs by increasing the voltage (overloading) and/or the amount of LEDs will probably not result in photoinhibition and the released photons may penetrate deeper into highly concentrated algal cultures, increasing biomass productivity with lower electrical power consumption [8]. However, both dimming methods can shift $\lambda_{\mathrm{e}}$ by $\sim 10 \mathrm{~nm}[77,78]$ and may change the photon capture when these peaks move towards or away from the optimal LHP absorption range, thus affecting microalgal growth. It is questionable which dimming method produces the highest biomass per input energy, because both have the aforementioned advantages and disadvantages. High-frequency PWM with AM-controlled LEDs may be implemented to reduce wavelength shifts and combine the advantages of both methods [78].

microalgae at lower PPFD with lower energy consumption could be possible if tailored light sources are selected for specific microalgal strains, growth phases, or photobioreactors.

\section{Concluding remarks and future perspectives}

Over the last few years, the prices of LED-based lamps have significantly decreased; nevertheless, LED lighting systems are still four times more expensive than FLs on average. However, this higher initial cost can be offset by the longer lifetimes and often better energy efficiency of LEDs compared to FLs. LED lighting technology is thus a feasible option for microalgal cultivation, in particular when supplemental light is needed for faster production of biomass and accumulation of specific biochemical components. As a result, research on microalgae grown under LEDs of different colors has strongly increased over the last 2-3 years. However, the combined application of different LEDs has seldom been studied, especially for LEDs emitting between 500 and $630 \mathrm{~nm}$ or above $700 \mathrm{~nm}$ (far-red region). Unfortunately, in many studies that used white LEDs, information about the emission spectra used is rarely mentioned, so meaningful comparisons are not possible. Moreover, optical density measurements for biomass determination in experiments with microalgae under different light qualities might be prone to error because the cell size and pigment composition can strongly change with light quality. Measurements of the PPFD of LEDs with narrow bandwidths using quantum sensors may also lead to errors because the responses of these sensors vary across the photosynthetically active range $(400-700 \mathrm{~nm})$. Thus, research on the application of LEDs in microalgal production should use properly calibrated spectroradiometers whenever feasible. Despite these problems, future research on LED use is promising and may lead to higher energy efficiency for indoor microalgal cultivation and improved reliability of research on the light requirements of biological and molecular processes in photosynthetic organisms. Furthermore, cultivation of microalgae in a highly energy-efficient manner can be envisaged using dimmed LEDs controlled by a combination of high-frequency PWM and amplitude modulation (AM) (Box 3). This application would require a greater number of LEDs to maintain the PPFD needed for cultivation. Nevertheless, because LEDs are not fully exploited when dimmed, heat generation is reduced, prolonging the lifetime of LED modules and possibly rendering LED cooling unnecessary, which are two critical factors for successful application of LEDs in microalgal production.

\section{Acknowledgments}

We wish to thank Prof. Rui Guerra from the Physics Department of the University of Algarve for his support in providing information and reviewing the manuscript.

\section{Appendix A. Supplementary data}

Supplementary data associated with this article can be found, in the online version, at http://dx.doi.org/10.1016/j.tibtech.2014.06.001.

\section{References}

1 Blanken, W. et al. (2013) Cultivation of microalgae on artificial light comes at a cost. Algal Res. 2, 333-340

2 Carvalho, A.P. et al. (2011) Light requirements in microalgal photobioreactors: an overview of biophotonic aspects. Appl. Microbiol. Biotechnol. 89, 1275-1288

3 Olle, M. and Virsile, A. (2013) The effects of light-emitting diode lighting on greenhouse plant growth and quality. Agric. Food Sci. $22,223-234$

4 US DOE (2013) Solid-State Lighting Research and Development. MultiYear Program Plan, Office of Energy Efficiency \& Renewable Energy, United States Department of Energy

5 Zhao, Y. et al. (2013) Effects of various LED light wavelengths and intensities on microalgae-based simultaneous biogas upgrading and digestate nutrient reduction process. Bioresour. Technol. 136, 461-468

6 Das, P. et al. (2011) Enhanced algae growth in both phototrophic and mixotrophic culture under blue light. Bioresour. Technol. 102, 38833887

$7 \mathrm{Fu}, \mathrm{W}$. et al. (2013) Enhancement of carotenoid biosynthesis in the green microalga Dunaliella salina with light-emitting diodes and adaptive laboratory evolution. Appl. Microbiol. Biotechnol. 97, 23952403

8 Lunka, A.A. and Bayless, D.J. (2013) Effects of flashing light-emitting diodes on algal biomass productivity. J. Appl. Phycol. 25, 1679-1685

9 Yan, C. et al. (2013) Effects of various LED light wavelengths and intensities on the performance of purifying synthetic domestic sewage by microalgae at different influent C/N ratios. Ecol. Eng. 51, 24-32

10 Kommareddy, A.R. and Anderson, G.A. (2003) Study of light as a parameter in the growth of algae in a photo-bioreactor (PBR). In ASAE International Meeting. ASAE Paper No. 034057

11 Pilon, L. et al. (2011) Radiation transfer in photobiological carbon dioxide fixation and fuel production by microalgae. J. Quant. Spectrosc. Radiat. 112, 2639-2660

12 Keeling, P.J. (2013) The number, speed, and impact of plastid endosymbioses in eukaryotic evolution. Annu. Rev. Plant Biol. 64, 583-607

13 Takaichi, S. (2011) Carotenoids in algae distribution, biosyntheses and functions. Mar. Drugs 9, 1101-1118 
14 Marriott, M.F.H. and Blankenship, R.E. (2011) Evolution of photosynthesis. Annu. Rev. Plant Biol. 62, 515-548

15 Itoh, K-I. et al. (2014) The influence of wavelength of light on cyanobacterial asymmetric reduction of ketone. Tetrahedron Lett. 55 , 435-437

16 Chen, H.B. et al. (2010) Modelling on chlorophyll $a$ and phycocyanin production by Spirulina platensis under various light-emitting diodes. Biochem. Eng. J. 53, 52-56

17 Tomitani, A. et al. (1999) Chlorophyll $b$ and phycobilins in the common ancestor of cyanobacteria and chloroplasts. Nature 400, 159-162

$18 \mathrm{Xu}$, B. et al. (2013) The effect of varying LED light sources and influent carbon/nitrogen ratios on treatment of synthetic sanitary sewage using Chlorella vulgaris. World J. Microbiol. Biotechnol. 29, 1289-1300

$19 \mathrm{Shu}, \mathrm{C} . \mathrm{H}$. et al. (2012) Effects of light on the accumulation of oil in a mixed culture of Chlorella sp. and Saccharomyces cerevisiae. J. Chem. Technol. Biotechnol. 87, 601-607

20 Beltran, L.M. et al. (2013) Web control and monitoring system: experimentation with Haematococcus pluvialis. Int. J. Eng. 26, 219-228

$21 \mathrm{Ho}$, S-H. et al. (2014) Enhancing lutein productivity of an indigenous microalga Scenedesmus obliquus FSP-3 using light-related strategies. Bioresour. Technol. 152, 275-282

22 Kim, T.H. et al. (2013) The effects of wavelength and wavelength mixing ratios on microalgae growth and nitrogen, phosphorus removal using Scenedesmus sp. for wastewater treatment. Bioresour. Technol. 130, 75-80

23 Abiusi, F. et al. (2014) Growth, photosynthetic efficiency, and biochemical composition of Tetraselmis suecica F\&M-M33 grown with LEDs of different colors. Biotechnol. Bioeng. 111, 956-964

24 Rendón, S.M. et al. (2013) Effect of carbon dioxide concentration on the growth response of Chlorella vulgaris under four different LED illumination. Int. J. Biotechnol. Wellness Ind. 2, 125-131

25 Baba, M. et al. (2012) Wavelength specificity of growth, photosynthesis, and hydrocarbon production in the oil-producing green alga Botryococcus braunii. Bioresour. Technol. 109, 266-270

26 Katsuda, T. et al. (2004) Astaxanthin production by Haematococcus pluvialis under illumination with LEDs. Enzyme Microb. Technol. 35, $81-86$

27 Grimm, B. et al., eds (2006) Chlorophylls and Bacteriochlorophylls: Biochemistry, Biophysics, Functions and Applications (Advances in Photosynthesis and Respiration, Vol. 25), Springer

28 Cheng, Y.C. and Fleming, G.R. (2009) Dynamics of light harvesting in photosynthesis. Annu. Rev. Phys. Chem. 60, 241-262

29 Scholes, G.D. et al. (2011) Lessons from nature about solar light harvesting. Nat. Chem. 3, 763-774

30 Zeinalov, Y. and Maslenkova, L. (2000) On the action spectra of photosynthesis and spectral dependence of the quantum efficiency. Bulg. J. Plant Physiol. 26, 58-69

31 Tennessen, D.J. et al. (1995) Efficiency of photosynthesis in continuous and pulsed light emitting diode irradiation. Photosynth. Res. 44,261-269

32 Koc, C. et al. (2013) Use of red and blue light-emitting diodes (LED) and fluorescent lamps to grow microalgae in a photobioreactor. Isr. J. Aquac. 65, 797 IJA_65 2013

33 Lee, C.G. and Palsson, B.Ø. (1996) Photoacclimation of Chlorella vulgaris to red light from light-emitting diodes leads to autospore release following each cellular division. Biotechnol. Prog. 12, 249-256

34 Saavedra, M.P.S. et al. (1996) Far-red light inhibits growth but promotes carotenoid accumulation in the green microalga Dunaliella bardawil. Physiol. Plant 98, 419-423

35 Stadnichuk, I.N. et al. (2011) Far-red light-regulated efficient energy transfer from phycobilisomes to photosystem I in the red microalga Galdieria suphuraria and photosystems-related heterogeneity of phycobilisome population. Biochim. Biophys. Acta 1807, 227-235

36 Tamulaitis, G. et al. (2005) High-power light-emitting diode based facility for plant cultivation. J. Phys. D: Appl. Phys. 38, 3182-3187

37 Bussell, A.N. and Kehoe, D.M. (2013) Hierarchical regulation between four-color and two-color cyanobacteriochromes. Proc. Natl. Acad. Sci. U.S.A. $110,12834-12839$

38 Kianianmomeni, A. and Hallmann, A. (2014) Algal photoreceptors in vivo functions and potential applications. Planta 239, 1-46

39 Beel, B. et al. (2012) A flavin binding cryptochrome photoreceptor responds to both blue and red light in Chlamydomonas reinhardtii. Plant Cell 24, 2992-3008
40 Lin, C. (2002) Blue light receptors and signal transduction. Plant Cell $14,207-225$

41 Ruyters, G. (1984) Effects of blue light on enzymes. In Blue Light Effects in Biological Systems (Senger, H., ed.), pp. 283-301, Springer

42 Costa, B.S. et al. (2013) Blue light is essential for high light acclimation and photoprotection in the diatom Phaeodactylum tricornutum. J. Exp. Bot. 64, 483-493

43 Kamiya, A. and Saitoh, T. (2002) Blue-light-control of the uptake of amino acids and of ammonia in Chlorella mutants. Physiol. Plant 116 , $248-254$

44 Marchetti, J. et al. (2013) Effects of blue light on the biochemical composition and photosynthetic activity of Isochrysis sp. (T-iso). J. Appl. Phycol. 25, 109-119

45 Giráldez, N. et al. (2000) Limiting $\mathrm{CO}_{2}$ levels induce a blue lightdependent $\mathrm{HCO}_{3}{ }^{-}$uptake system in Monoraphidium braunii. J. Exp. Bot. 51, 807-815

46 Pazos, J.V.P. and Izquierdo, P.F. (2011) Synthesis of neutral lipids in Chlorella sp. under different light and carbonate conditions. Cien. Tecnol. Fut. 4, 47-58

47 Jahns, P. and Holzwarth, A.R. (2012) The role of the xanthophyll cycle and of lutein in photoprotection of photosystem II. Biochim. Biophys. Acta 1817, 182-193

48 Depauw, F.A. et al. (2012) Exploring the molecular basis of responses to light in marine diatoms. J. Exp. Bot. 63, 1575-1592

49 Kim, H.H. et al. (2004) Green-light supplementation for enhanced lettuce growth under red- and blue-light-emitting diodes. Hortscience 39, 1617-1622

50 Terashima, I. et al. (2009) Green light drives leaf photosynthesis more efficiently than red light in strong white light revisiting the enigmatic question of why leaves are green. Plant Cell Physiol. 50, 684-697

51 Mohsenpour, S.F. and Willoughby, N. (2013) Luminescent photobioreactor design for improved algal growth and photosynthetic pigment production through spectral conversion of light. Bioresour. Technol. 142, 147-153

52 Tsao, J.Y. et al. (2009) Solid-state lighting an integrated human factors, technology and economic perspective. Proc. IEEE 98, 1-17

53 Kane, R. et al., eds (2002) Revolution in Lamps: A Chronicle of 50 Years of Progress (2nd edn), Fairmount Press

54 Crawford, M.H. (2009) LEDs for solid-state lighting performance: challenges and recent advances. IEEE J. Sel. Top. Quant. Electron. $15,1028-1040$

55 Chen, Y.C. and Lee, M.C. (2012) Double-power double heterostructure light-emitting diodes in microalgae Spirulina platensis and Nannochloropsis oculata, cultures. J. Mar. Sci. Technol. 20, 233-236

56 Park, Y.G. et al. (2012) Light source and $\mathrm{CO}_{2}$ concentration affect growth and anthocyanin content of lettuce under controlled environment. Hortic. Environ. Biotechnol. 53, 460-466

57 Tang, H. et al. (2011) Culture of microalgae Chlorella minutissima for biodiesel feedstock production. Biotechnol. Bioeng. 108, 2280-2287

58 Müller-Mach, R. et al. (2009) All-nitride monochromatic amberemitting phosphor-converted light-emitting diodes. Phys. Status Solidi 3, 215-217

59 Yan, C. et al. (2013) Effects of various LED light wavelengths and light intensity supply strategies on synthetic high-strength wastewater purification by Chlorella vulgaris. Biodegradation 24, 721-732

60 Yan, C. and Zheng, Z. (2014) Performance of mixed LED light wavelengths on biogas upgrade and biogas fluid removal by microalga Chlorella sp. Appl. Energy 113, 1008-1014

61 Paradiso, R. et al. (2011) Light use efficiency at different wavelengths in rose plants. Acta Hortic. 893, 849-856

62 Grobbelaar, J.U. et al. (1996) Influence of high frequency light/dark fluctuations on photosynthetic characteristics of microalgae photoacclimated to different light intensities and implications for mass algal cultivation. J. Appl. Phycol. 8, 335-343

63 Matthijs, H.C.P. et al. (1996) Application of light-emitting diodes in bioreactors: flashing light effects and energy economy in algal culture (Chlorella pyrenoidosa). Biotechnol. Bioeng. 50, 98-107

64 Redwood, M.D. et al. (2012) Enhanced photosynthetic output via dichroic beam-sharing. Biotechnol. Lett. 34, 2229-2234

$65 \mathrm{Wu}$, D. et al. (2013) Algae (Microcystis and Scenedesmus) absorption spectra and its application on chlorophyll $a$ retrieval. Front. Earth Sci. $7,522-530$ 
66 Coltelli, P. et al. (2013) Automatic and real time recognition of microalgae by means of pigment signature and shapes. Environ. Sci. Process. Impacts 15, 1397-1410

67 Nhut, D.T. and Nam, N.B. (2010) Light-emitting diodes (LEDs): an artificial lighting source for biological studies. IFMBE Proc. 27, 134-139

68 Elmoraghy, M. and Farag, I.H. (2012) Bio-jet fuel from microalgae reducing water and energy requirements for algae growth. Int. J. Eng. Sci. 1, 22-30

69 Miao, H. et al. (2012) Study on the effect of monochromatic light on the growth of the red tide diatom Skeletonema costatum. Opt. Photonics J. $2,152-156$

70 Ross, J. and Sulev, M. (2000) Sources of errors in measurements of PAR. Agric. For. Meteorol. 100, 103-125

71 Barnes, C. et al. (1993) Accuracy of quantum sensors measuring yield photon flux and photosynthetic photon flux. Hortic. Sci. 28, 1197-1200

72 Yoshioka, M. et al. (2012) Effects of high frequency of intermittent light on the growth and fatty acid profile of Isochrysis galbana. Aquaculture 338-341, 111-117
73 Chen, C-Y. et al. (2013) Engineering strategies for enhancing the production of eicosapentaenoic acid (EPA) from an isolated microalga Nannochloropsis oceanica CY2. Bioresour. Technol. 147, $160-167$

74 Kwon, H.K. et al. (2013) Laboratory study for the phytoremediation of eutrophic coastal sediment using benthic microalgae and light emitting diode (LED). J. Fac. Agric. Kyushu Univ. 58, 417-425

75 Mynbaev, D.K. and Scheiner, L.L., eds (2006) Fiber-Optic Communications Technology, Prentice Hall

76 Miller, D.A.B. et al. (1996) Optical physics of quantum wells. In Quantum Dynamics of Simple Systems (Oppo, G.-L. et al., eds), pp. 239-266, Institute of Physics

$77 \mathrm{Gu}$, Y.et al. (2006) Spectral and luminous efficacy change of high-power LEDs under different dimming methods. Proc. SPIE 6337, 63370J

78 Beczkowski, S. and Nielsen, S.M. (2010) LED spectral and power characteristics under hybrid PWM/AM dimming strategy. In Proceedings of the 2010 Energy Conversion Congress and Exposition. pp. 731-735, IEEE Press 